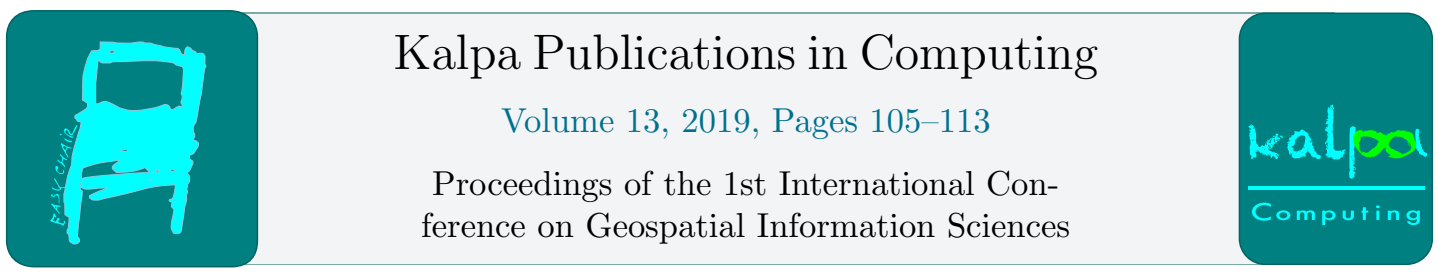

\title{
A review on the color segmentation with emphasis on the point clouds
}

\author{
Sergio Macías-Medrano \\ CentroGeo Campus CDMX, México.
}

\begin{abstract}
Currently, there are technologies such as Unmanned Aerial Vehicles (UAV) that allow more efficient field work in geology. With the capture of aerial photographs and their processing with algorithms based in Structure from Motion (SfM), it is possible to obtain a position XYZ with their RGB color. The resulting outputs, whether images or point clouds, can be used to recognize patterns of geological layer in stratified walls from color. This semi-automatic segmentation allows to identify subtle changes between lithologies through the transformation of RGB to other color spaces such as CIELab.

The review was done around the issues of color segmentation identified through a search level scheme. These were color information, type of spatial data, segmentation methods and color spaces.
\end{abstract}

\section{Introduction}

Obtaining 3D point clouds of high accuracy represents an important source of information for Earth Sciences studies. Although the generation of this type of spatial data can be carried out with several technologies such as Terrestrial Laser Scanner (TLS) or SfM.

Point clouds derived from the SfM process have color information in the RGB space, which makes it possible the color segmentation, however, the main problem is the projection of shadows and obstacles such as vegetation.

The outputs derived from the TLS, are obtained by measurement of the wavelength of the beam of light emitted and received. It is possible to filter the vegetation and recognize geological layers by obtaining values. In this sense, Humair et al. (2015) mentions that it is necessary to consider elements such as the composition of the material, the distance and the angle of incidence with respect to the object, among others, which can be represented a problem in the process of correct TLS intensity values.

The benefits offered by photogrammetry from UAV are suitable for a teaching environment, where there is not a lot of economic resources to acquire high-cost technological equipment, however, it does not mean that the results are unreliable. 
Through the SfM algorithm, which starts from photogrammetry and developed with the computer vision systems, it is possible to generate a restitution from photographs overlapped on each other, of a real object, for a digital 3D visualization of it (Fonstad et al., 2013).

Thus, geology finds an area of opportunity that is constantly expanding with the use of point clouds for the generation of knowledge related to structural geology or rock mechanics (Riquelme et al., 2014). In addition, by opting for the use of UAV, allows the Earth Sciences specialist to remotely approach inaccessible areas. This is the case of study of rock walls that can be unstable or have great magnitudes that could affect the analysis in its fullness with conventional techniques.

The objective is segmenting the lithologies of a wall of stratified rocks, from a point cloud obtained with aerial photographs processed with Colmap SfM algorithms. Due to the flexibility of $\mathrm{UAV}$, it is possible to develop photogrammetric projects with a horizontal perspective, that is, perpendicular to the axis of the wall and specify flight lines from destination on the base axis emulating a project terrestrial photogrammetry (Caire, 1977), where only the flight height for each line is changed in order to cover the entire wall. In addition, the RGB color provided by the camera mounted to the UAV can be an input for the identification of the lithologies.

The reviewed papers were focused in the color point cloud segmentation derived from the SfM process. The above, since the presence of color in lithologies is a characteristic that, in principle, makes it possible to identify them in the field by specialist naked eye, so it is sought to find a method that facilitates this task through geospatial information technologies, considering that UAV allow the development of photogrammetric projectsto guarantee a suitable product. However, it is not a trivial task, since they must consider several elements that are intrinsic to the point clouds derived from the SfM process such as vegetation or shadows at the time of photographs acquisition and that may affect the interpretation of color. In this sense, the aim question is if it is possible to adapt analogous methodologies of color point cloud segmentation to the identification of lithologies.

\section{Materials and methods}

\subsection{Paper search Paper selection}

The searches were carried out through several levels, starting from the particular to the general. This means that the first level consisted in looking for what has been said regarding the segmentation of lithologies by color, hence the definition of a lithology segmentation point cloud and its derivatives to enter the engines of search, mainly motivated by the base studies (Humair et al., 2015; Vasuki et al. , 2017) that are the guide at the beginning of the research project for which this bibliographic review is carried out.

As a second level of search, the review of works cited in the two base studies was carried out, those related to the segmentation of point clouds.

In the third level, the search was more general through keywords such as color segmentation, intensity segmentation, monochromatic intensity and point cloud segmentation. The purpose of this level was to find analogous methodologies that defined in the research.

\subsection{Paper search Paper selection}

As a result of the search process by levels, a set of preliminary elements was obtained that should have been previously classified by thematic folders and evaluated to define their relevance in the context of color segmentation by means of point clouds.

In the reading of each one, several aspects were considered, among others the research question, starting data and methods used. 
The theory related to color spaces was a relevant topic at the time of the selection of the articles, especially those that indicate the characteristics, weaknesses and aptitudes when considering a segmentation by color.

\subsection{Comparison method}

A comparative table is presented focused on describing in general the algorithms used by the different works, which defines the authors, application, type of spatial data, source of information, segmentation method, input/color space. Thus, it will be possible to build a tool for the discussion of the different proposals around color segmentation.

The development of the main body is fed back from the analysis, contrast and development of ideas derived from the table of comparison of methods related to the structure of the type of spatial data, relationship of type of spatial data with segmentation methods and relationship of segmentation methods with input/color space.

\section{Color segmentation}

In general terms, color segmentation has been a subject studied for many years, which allows a solid theoretical framework. It is approached from two different types of spatial data: images (Bali \& Singh, 2015; Katiyar \& Chitade, 2010; Rodríguez-Pulido, et al., 2013) and point clouds (Sapkota, 2008; Xu et al., 2019; Zhan et al., 2009).

\subsection{Color information}

Using the images to group certain characteristic elements has a great development in segmentation starting from monochromatic values, the histogram threshold, identification of characteristic forms or detection of edges, among others, based on discontinuity or homogeneity of the gray scale values.

Cheng et al. (2001), points out that using color in place of monochromatic scale allows maximizing the results, where clustering in a single band can be done through the combination of the red (R), green $(\mathrm{G})$ and blue (B) components, with which the human perceives the color. While RGB color space is the most common, it is not the only one, since there are many others such as LAB, HSV and LUV that are used for segmentation.

The RGB color space can be represented through three dimensions with axes ranging from 0 to 255 , so that the coordinates of a point represent the values of each component that together define a color. This point is in accordance with the laws of colorimetry, which define the following:

1) Any color can be created by these three colors and the combination of the three is unique; 2) if two colors are equivalent, they will be equivalent again after multiplying or dividing the three components by the same number; 3) The luminance of a combination of colors is equivalent to the sum of the luminance of each color (Chapron, 1992)

The interpretation of the first law under the context of lithology segmentation, indicates that the color of these is a first approximation for its identification and labeling in the field, which can be done directly from a model such as the point cloud or the image, although always taking into account the effect of the shadows.

Although the RGB space is adequate to visualize the color, it is not suitable for segmentation, since there is a high correlation between the three components, that is, if the intensity of the color changes, the three components will do so, according to the third law of colors. In addition, the analysis of the similarity between two colors is not feasible, since the measurement of a color does not 
represent color differences on a uniform scale (Cheng et al., 2001). This implies having to make transformations between color spaces that are divided between linear (YIQ or YUV) and nonlinear (HSI, CIE or Munsell).

In the case of the CIE color space, it is characterized by having three elements: XYZ. This space can be the product of a linear transformation from the RGB coordinates, as it is shown in the following:

$$
\left(\begin{array}{l}
X \\
Y \\
Z
\end{array}\right)=\left(\begin{array}{lll}
0.607 & 0.174 & 0.200 \\
0.299 & 0.587 & 0.114 \\
0.000 & 0.066 & 1.116
\end{array}\right)\left(\begin{array}{l}
R \\
G \\
B
\end{array}\right)
$$

There are variants of the CIE space, once the XYZ elements are already known. An example of this is CIELab that aggregates the components L, a and b (Katiyar \& Chitade, 2010), obtained from a non-linear transformation of the $\mathrm{XYZ}$ values:

$$
\begin{array}{r}
L=116\left(\sqrt[3]{\frac{Y}{Y_{0}}}\right)-16 \\
a=500\left[\sqrt[3]{\frac{X}{X_{0}}}-\sqrt[3]{\frac{Y}{Y_{0}}}\right] \\
b=200\left[\sqrt[3]{\frac{Y}{Y_{0}}}-\sqrt[3]{\frac{Z}{Z_{0}}}\right]
\end{array}
$$

where $\mathrm{Y} / \mathrm{Y} 0>0.01, \mathrm{X} / \mathrm{X} 0>0.01$ and $\mathrm{Z} / \mathrm{Z} 0>0.01$, while $\mathrm{X} 0, \mathrm{Y} 0$, and $\mathrm{Z} 0$ are $\mathrm{X}, \mathrm{Y}$ and $\mathrm{Z}$ values for the standard white (Cheng et al., 2001).

The virtue of this variant is that each point represents a color in space that has the possibility of being differentiated from another through the euclidean distance between the two points, even when the visual difference is barely detectable. Because of these characteristics, the color space CIELab is currently recommended by the International Commission on Illumination (Rodríguez-Pulido et al., 2013).

CIELab is a method that has been used for applications related to the detection of lithologies, since one of its characteristics is the representation of color through luminousness (Vasuki et al., 2017). This allows to work with the segmentation of a lithology by its color even when the change in hue is subtle, since the euclidean distance between these two points can be calibrated to decide whether it represents the same element or not.

A valuable contribution that leaves the work of Cheng et al. (2001), lies in the discussion about the transformations. He points out that one of the biggest problems of transforming linearly to color space is found to have high correlation between the three RGB components, as mentioned above. This translates into a difficulty in discrimination of color and shadow enhancement. It must be remembered that, if we work on color segmentation in a linear space, we will use a space in three dimensions that translate it into a component. In contrast, nonlinear transformation methods can work in one dimension, such as the HSI space, to rule out certain shadows that could affect information processing.

\subsection{Table}

The following is a comparative table that aims to gather the most relevant characteristics of the researches consulted on color segmentation, according to the parameters established in this paper. 


\begin{tabular}{|c|c|c|c|c|c|}
\hline Author & Application & $\begin{array}{l}\text { Type of } \\
\text { spatial } \\
\text { data }\end{array}$ & $\begin{array}{l}\text { Source of } \\
\text { information }\end{array}$ & $\begin{array}{l}\text { Segmentation } \\
\text { method }\end{array}$ & $\begin{array}{l}\text { Input/ } \\
\text { Color space }\end{array}$ \\
\hline $\begin{array}{c}\text { Katiyar \& } \\
\text { Chitade, } \\
2010\end{array}$ & $\begin{array}{c}\text { Color } \\
\text { segmentation } \\
\text { of satellite } \\
\text { images }\end{array}$ & Image & $\begin{array}{c}\text { Images of } \\
\text { Cartosat-1 and } \\
\text { LISS-III }\end{array}$ & K-Means & CIELab \\
\hline $\begin{array}{l}\text { Humair et } \\
\text { al., } 2015\end{array}$ & $\begin{array}{l}\text { Detection of } \\
\text { geological } \\
\text { layers }\end{array}$ & $\begin{array}{l}\text { Point } \\
\text { cloud }\end{array}$ & $\begin{array}{c}\text { TLS and } \\
\text { Terrestrial } \\
\text { Photogrammetry }\end{array}$ & $\begin{array}{l}\text { Connected- } \\
\text { component } \\
\text { labeling }\end{array}$ & $\begin{array}{c}\text { Intensity of LiDAR } \\
\text { and RGB Blue } \\
\text { Monochromatic } \\
\text { Band }\end{array}$ \\
\hline $\begin{array}{l}\text { Rodríguez- } \\
\text { Pulido et al., } \\
2013\end{array}$ & $\begin{array}{l}\text { Analysis of } \\
\text { the } \\
\text { appearance } \\
\text { of the fruit }\end{array}$ & Image & Photography & Fuzzy & CIELab \\
\hline $\begin{array}{l}\text { Sapkota, } \\
2008\end{array}$ & $\begin{array}{c}\text { Segmentation } \\
\text { of } \\
\text { information } \\
\text { derived from } \\
\text { unstructured } \\
\text { laser point } \\
\text { clouds }\end{array}$ & $\begin{array}{l}\text { Point } \\
\text { cloud }\end{array}$ & TLS & $\begin{array}{l}\text { Hybrid } \\
\text { (Region- } \\
\text { growing }+ \\
\text { color) }\end{array}$ & CIELab \\
\hline $\begin{array}{l}\text { Vasuki et } \\
\text { al., } 2017\end{array}$ & $\begin{array}{l}\text { Detection of } \\
\text { lithological } \\
\text { edges }\end{array}$ & Image & Photography & $\begin{array}{c}\text { Interactive } \\
\text { clustering } \\
\text { (Superpixel + } \\
\text { Region- } \\
\text { growing) } \\
\end{array}$ & CIELab \\
\hline $\begin{array}{c}\text { Xu et al., } \\
2019\end{array}$ & $\begin{array}{c}\text { Detection of } \\
\text { workpieces } \\
\text { in the } \\
\text { industry }\end{array}$ & Image & Kinect V2 & $\begin{array}{c}\text { Convolutional } \\
\text { neural network }\end{array}$ & $\begin{array}{l}\text { RGB and image } \\
\text { depth }\end{array}$ \\
\hline $\begin{array}{l}\text { Zhan et al., } \\
2009\end{array}$ & $\begin{array}{l}\text { Segmentation } \\
\text { of complex } \\
\text { architectural } \\
\text { forms } \\
\end{array}$ & $\begin{array}{l}\text { Point } \\
\text { cloud }\end{array}$ & TLS & $\begin{array}{l}\text { Hybrid } \\
\text { (Region- } \\
\text { growing }+ \\
\text { color) } \\
\end{array}$ & $\begin{array}{c}\text { Vector normal y } \\
\text { RGB }\end{array}$ \\
\hline Zhan et al., & $\begin{array}{l}\text { Segmentation } \\
\text { of surfaces } \\
\text { hv } n \text { nnrmal }\end{array}$ & Point & TI $\mathrm{C}$ & $\begin{array}{l}\text { Hybrid } \\
\text { (Region- }\end{array}$ & Vector normal y \\
\hline
\end{tabular}

\subsection{Color image segmentation}

The image has the characteristic of being a structured 2D model with an array of cells as a matrix, where each one has its neighboring pixels defined.

Comparison type of spatial data versus segmentation method:

Image segmentation is a process that seeks homogeneity through its characteristics that are 
connected by means of pixels (Cheng et al. 2001) and subsequently labeled in different classes. This process is known as image survey or image analysis that allows the object and background to be separated (Bali \& Sing h, 2015). Through a comparison between methods, there is no one that is perfect, since segmentation depends on multiple factors such as color, texture, intensity and uniformity of the image.

That is why, as regards the recognition of color patterns, there are many proposals for procedures that must be classified in principle among methods of monochromatic segmentation in gray scale and color. Cheng et al. (2001) defined for the first case clustering, edge of detection and region extraction. However, it should be mentioned that the color image provides more information and is useful for pattern recognition. In this sense, the color image is considered as multi-spectral and has two large groups of color segmentation: boundary formation and region formation.

In the table, the methods of color segmentation that address the problem from different visions are retaken. Vasuki et al. (2017) propose a method of delimitation of lithologies based on the segmentation of superpixels that with the help of the region-growing process, pixels can be clustered. While Katiyar and Chitade (2010) apply a general methodology based on clustering segmentation applied to satellite images to identify $\mathrm{K}$ numbers of color patterns through the K-means algorithm. Rodríguez-Pulido et al. (2013) part of which each point is a potential cluster center and calculates a measure of likelihood for each point that can be a cluster center. Finally, Xu et al. (2019) develop the classification of workpieces in the industry with the convolutional neural network method, which works based on training the algorithm from images.

Comparison segmentation method versus Input/Color space:

In the case of three methods that uses image segmentation, the CIELab color space coincides because it is the most appropriate when distinguishing the color difference between two pixels by means of a euclidean distance measurement, so any of the color segmentation methods proposed in these studies can be adapted to the classification of pixels by means of CIELab. While the convolutional neural network algorithm recognizes images of workpieces that are acquired by a Kinect that recognizes image depth and RGB color.

\subsection{Color point cloud segmentation}

The point cloud is a type of $3 \mathrm{D}$ data that is presented as unstructured, which means that a point has no defined neighbors. To know the relationship between its adjacent elements, it is necessary to use operations from closer neighbors (Grilli e t al., 2017).

Comparison type of spatial data versus segmentation method:

Unlike the segmentation of images, the segmentation of point clouds starts in the first instance of criteria of spatial proximity and geometric properties as the normal vector to clustering points. It is a process of labeling each point within the point cloud and its subsequent clustering considering the properties according to the defined objectives.

There are three large clustering groups (Zhan et al., 2010), based on: edges, surfaces or hybrid. The algorithms of segmentation edges give an account of the forms based on the normal vector, gradients and curvatures, in this way all that point that is within an edge that delimits these forms, will be clustered in the same class. In the case of surface segmentation, a homogeneous continuous plane or with similar geometric properties is detected, one of these methods is the region- growing. Finally, for the hybrid segmentation it is based on several properties of the points that can measure the geometry, color or texture. 
As can be seen in Table 1, three of the four color point cloud segmentation methods use the region-growing method based on surface segmentation, which is developed in the works of Zhan et al. $(2010,2009)$ and Sapkota (2008). In the case of the first two articles, the application focuses on the detection of the different architectural surfaces. Part of segment normal vectors that represent different planes, through which a color is assigned to each vector. With a previous normalization of the vector component, it is transformed linearly to the RGB component, this allows segmenting the surfaces by color and identifying the different elements that exist within the data set. For this reason, finally the two methods proposed by Zhan, are hybrids.

In the case of Sapkota (2008), the motivation of his study lies in the identification of surfaces, which he intends to segment through the region-growing method in conjunction with the color information. To make the region-growing, part of a seed surface that combines the criteria of proximity, planarity and surface smoothness.

Humair et al. (2015) propose a different methodology focused on the identification of lithologies through color. To do this, they use the connected-component labeling method to relate regions of points. This means having previously segmented from a thresholding or cluster defined by the median and deviation standard of intensity values.

Comparison segmentation method versus Input/Color space:

Previously, it was commented that CIELab color space is more efficient than RGB, since it works with its components in an independent way and elements with subtle color changes can be identified more easily. However, when working on the region-growing method to identify surfaces through the normal vector (see table 1), from which a transformation is made to a color space so that the identified surface can be extended, it is useful to work with RGB, since the conversion is linear and the segmentation is supported by both the normal vector and the color.

However, there are applications of the region-growing process where it is not enough to use the RGB color space, especially when it is intended to identify surfaces from a seed plane together with a criterion of similarity in color.

On the other hand, Sapkota (2008) relies on the kd tree data structure to provide the explicit neighborhood relationship for each point. With the neighborhood index created it is possible to identify patterns by color, so the next step is to transform the RGB from CIELab to avoid shading and to measure euclidean distance.

In the case of the work of Humair et al. (2015) the application is related to the detection of lithologies through the monochromatic channel in the blue band from the RGB. This means that the brightness of said channel is represented in 8-bit grayscale $(0-255)$. In this way, thresholds can be defined for each lithology and subsequently regions from the connected-component labeling method.

\section{Summary}

As for the first level search, it was not possible to obtain an acceptable result, since it is a very specific topic. Most of the articles of this level related to the segmentation of lithologies that were part of the set of preliminary elements, are about segmentation by means of the intensity derived from the information of the TLS, which belong to the second level of search. The decision not to include these articles was for two aspects, on the one hand, it is a methodology that is not contemplated for the investigation since the information that will be used will be from point clouds of a photogrammetric flight and on the other, the way in which the process works is attended by the research of Humair et al. (2015) at least in this first phase of state of the art aimed at color segmentation. 
Of the same work points to one aspect that remains, as it also uses the processing intensity obtained from monochrome blue band channel belonging to RGB space for segmenting lithologies. The origin of the point cloud is from terrestrial photogrammetry with a conventional RGB camera. This is an aspect that motivated the search more general to articles through the keywords intensity segmentation and monochromatic intensity, however, the results were derived from other aspects and no work was found in the first instance that could elucidate the subject.

The rest of the keywords belonging to the third level of search (color segmentation, color point cloud segmentation and point cloud segmentation) allow to have a context concerning topics of great importance. It should be mentioned that the search at this level considers color segmentation using both the image and point cloud.

Among the relevant aspects of this search is the discussion of the different color spaces and their transformations. In addition, it is shown in the comparative methods that the CIELab space is the most used when segmenting by color. This space of color allows measuring the euclidean distance between colors even when the shade is subtle.

In the case of the articles consulted that use color segmentation in the RGB space, they use a criterion of depth (image depth and normal vector) of the objects using segmentation methods, so, under these conditions, the RGB color space works properly.

The methodologies used are very varied, this is evident in the accompanying table and is reinforced in the literature consulted with a focus on reviewing progress and perspectives in two different periods with a laps of 20 years (Cheng et al., 2001) and 15 years (Bali \& Singh, 2015) approximately in the search.

\section{Conclusions}

The transformations between color spaces allow to increase the segmentation capacity to characterize different geometric aspects of the point clouds such as the edges or the normal vectors (Zhan et al. , 2010), and even the ages of the object of study such as case of the color or texture of lithologies (Vasuki et al., 2017).

The applications of color segmentation on point cloud models derived from photogrammetric processes with conventional cameras are still pending. There is a dominance in the use of TLS technology in contrast to photogrammetry for these purposes, however, the reason why this situation occurs remains to be discussed. In addition, there is evidence that the most commonly used type of spatial data for color segmentation are images.

The multiplicity of segmentation algorithms by means of color, accompanied by the justification of the selection of the methodology, as well as the favorable results in the evaluation methods indicate that the segmentation methods are suitable to characterize and group properties of the objects study.

Although, there is a previous work of segmentation by color of lithologies as is the case of Vasuki et al. (2017), this is directly using images for classification. In this sense, one of the remaining points is to perform this same work on point clouds. Although these are two different types of spatial data as defined above, they have characteristics in common, such as starting from an RGB color space, which is possible to transform other spaces into both one and another model. In this sense, the CIELab color space works properly when segmenting elements, even when they have subtle color changes.

This allows us to propose in an exploratory way the adaptation of analogous methodologies on color segmentation, despite being applied to images as well as point clouds, for the identification of lithologies using the products derived from the SfM algorithm.

Even when the structure of the type of spatial data is different, their methods segmentation shares theoretical concepts and algorithms that can propose a method for identification of lithologies through point clouds derived from the process SfM. 


\section{References}

Bali, A., \& Singh, SN (2015). A review on the strategies and techniques of image segmentation. In International Conference on Advanced Computing and Communication Technologies, ACCT. https://doi.org/10.1109/ACCT.2015.63

Caire, J. (1977). Photogrammetry-I (First Ed). Federal District: Rodriguez Editorial.

Chapron, M. (1992). A new chromatic edge detector used for color image segmentation. In Proceedings - International Conference on Pattern Recognition. https://doi.org/10.1109/ICPR.1992.201987

Cheng, HD, Jiang, XH, Sun, Y., \& Wang, J. (2001). Color image segmentation: Advances and prospects. Pattern Recognition. https://doi.org/10.1016/S0031-3203(00)00149-7

Fonstad, MA, Dietrich, JT, Courville, BC, Jensen, JL, \& Carbonneau, PE (2013). Topographic structure from motion: A new development in photogrammetric measurement. Earth Surface Processes and Landforms. https://doi.org/10.1002/eng.3366

Grilli, E., Menna, F., \& Remondino, F. (2017). A review of point clouds segmentation and classification algorithms. In International Archives of the Photogrammetry, Remote Sensing and Spatial Information Sciences - ISPRS Archives. https://doi.org/10.5194/isprs-archives-XLII-2-W3339-2017

Humair, F., Abellan, A., Carrea, D., Matasci, B., Epard, JL, \& Jaboyedoff, M. (2015). Geological layers detection and characterization using high resolution 3D point clouds: Example of a box-fold in the Swiss Jura Mountains. European Journal of Remote Sensing. https://doi.org/10.5721/EuJRS20154831

Katiyar, \& Chitade. (2010). Color based image segmentation using k-means clustering. International Journal of Engineering Science and Technology.

Riquelme, AJ, Abellán, A., Tomás, R., \& Jaboyedoff, M. (2014). A new approach for semi-automatic rock mass joints recognition from 3D point clouds. Computers and Geosciences. https://doi.org/10.1016/j.cageo.2014.03.014

Rodríguez-Pulido, FJ, Gordillo, B., Lourdes González-Miret, M., \& Heredia, FJ (2013). Analysis of food appearance properties by computer vision applying ellipsoids to color data. Computers and Electronics in Agriculture. https://doi.org/10.1016/j.compag.2013.08.027

Sapkota, PP (2008). Segmentation of colored point cloud data. International Institute for GeoInformation Science and Earth Observation.

Vasuki, Y., Holden, EJ, Kovesi, P., \& Micklethwaite, S. (2017). An interactive image segmentation method for lithological boundary detection: A rapid mapping tool for geologists. Computers and Geosciences. https://doi.org/10.1016/j.cageo.2016.12.001

Xu, H., Chen, G., Wang, Z., Sun, L., \& Su, F. (2019). RGB-D-based pose estimation of workpieces with semantic segmentation and point cloud registration. Sensors (Switzerland). https://doi.org/10.3390/s19081873

Zhan, Q., Yu, L., \& Liang, Y. (2010). A point cloud segmentation method based on vector estimation and color clustering. In 2nd International Conference on Information Science and Engineering, ICISE2010 - Proceedings. https://doi.org/10.1109/ICISE.2010.5691038

Zhan, Q., Yubin, L., \& Xiao, Y. (2009). Color-Based Segmentation of Point Clouds. Laser Scanning 2009, IAPRS. https://doi.org/XXXVIII-3/W8 